\title{
Psychological Impact on Sexual Health among Diabetic Patients: a Review
}

\author{
Muhammad A Siddiqui ${ }^{1}$, Zeeshan Ahmed ${ }^{2, *}$, Adeel Ahmed Khan ${ }^{3}$ \\ ${ }^{1}$ School of Health Sciences, Queen Margaret University, Edinburgh, UK \\ ${ }^{2}$ Department of Community Medicine, Dow University of Health Sciences, Karachi, Pakistan \\ ${ }^{3}$ Department of Community Health Sciences, Aga Khan University Hospital, Karachi, Pakistan
}

\begin{abstract}
The aim of this review is to discuss the psychological impact on sexual health among diabetic population and association of diabetes with sexual problems and psychological problems. Diabetes is known to cause multiple medical, psychological, and sexual dysfunctions. Impaired sexual function in men is a well-documented complication of diabetes. Although women have the same risk to develop diabetic complications. This review indicates that sexual problems cluster with self-reported physical problems in men, and with psychological and social problems in women. This has potentially important consequences for the planning of treatment for sexual problems and implies that effective therapy could have a broad impact on sexual health in the diabetic population.
\end{abstract}

Keywords Diabetes Mellitus, Sexual Health, Sexual Dysfunction, Erectile Dysfunction, Orgasmic Dysfunction

\section{Introduction}

Diabetes mellitus is a chronic and progressive diseases marked by hyperglycaemia resulting from inadequate production of insulin and insulin action, or both. It associated with long term serious complications and premature death, but people with diabetes can take steps to control the disease and lower the risk of complications ${ }^{1}$. The World Health Organization estimates that more than 180 million people worldwide have diabetes and this number is likely to more than double by $2030^{2}$. Anxiety and depression is a common psychological dilemma in diabetic patients. Diabetes is a common disorder; causes multiple medical, psychological and sexual dysfunctions. Impaired sexual function in men is a well-recognized complication of diabetes ${ }^{3}$.

Sexual problems (collapse of sexual function) secondary to diabetes was first described by Avicenna in 960-1037 $\mathrm{AD}^{4}$. Over the past century there have been several authors who confirm this observation. The past 70 years have seen increasingly rapid advances in the development of education about relationship in diabetes and sexual health. In early 1940s medical textbook recognize the sexual health as complication of diabetes. However, far too little attention was paid on female sexual dysfunction. In 1950s researchers has shown great interest to widen the knowledge of female sexuality and sexual dysfunction in men with diabetes ${ }^{5}$. In 1971 published first article published on influence of

* Corresponding author:

dr.zeeshan_jmch@yahoo.com (Zeeshan Ahmed)

Published online at http://journal.sapub.org/diabetes

Copyright (C) 2012 Scientific \& Academic Publishing. All Rights Reserved diabetes on female sexual health ${ }^{6}$.

Most studies of sexual dysfunction in diabetic men have focused on erectile dysfunction. However, the minority of studies discussed any other form of sexual dysfunction. Abnormal sexual health has consequences on the psychological well-being and reproductive function. They can be the first symptom of comorbidities or a treatment side effect. Erectile dysfunction is increasingly being recognised as an early marker of organic incipient systemic disease ${ }^{7}$. In contrast female sexual dysfunction has not been well studied among diabetic women. In women, neuropathy, vascular impairment, and psychological complaints have been implicated in the pathogenesis of decreased libido, low arousability, decreased vaginal lubrication, orgasmic dysfunction, and dyspareunia among diabetic women ${ }^{8}$. The prevalence of sexual dysfunction in diabetic men approaches 50\%, whereas in diabetic women it seems to be slightly lower ${ }^{9-11}$.

Health psychology aimed at, looking to the patient as a whole, instead of concentrating any particular problem coming out of the diabetes are concentrating toward the whole person. This includes, identifying the behavioural risk factors, behavioural maintenance of health, health/illness cognition, communication decision making and adherence to prescribed management, coping with illness and disability ${ }^{12}$. Some of the good outcomes of good communication are: increase in patient knowledge and recall, increase patient satisfaction, genuinely informed consent, increase patient compliance to treatment hence preventing unexpected complications and quicker recovery from illness.

The purpose of this paper is to review the pathophysiology, psychological quality of life effect, and management of sexual dysfunction in men and women with diabetes. 


\section{Discussion}

All forms of psychiatric disorder can lead to disturbances in sexuality, either directly, through common effects on the central nervous system, or indirectly, as a result of social or psychological changes and drugs' side effects ${ }^{13}$. A numbers of studies have looked at the relation between sexual problems and specific physical conditions. The most widely reported association is between male sexual problems and diabetes $^{14}$. However major problem with erectile dysfunction in men is psychological impact such as depression, anxiety and lower sexual efficacy ${ }^{15}$. Depressed subjects normally have a poorer quality of life (physical, psychological and social), independent of the physical illnesses from which they might suffer.

A study was conducted to examine the association of diabetes with sexual dysfunction. The result of trial concluded that diabetes significantly associated not only with erectile dysfunction but with all aspects of sexual dysfunction, including sexual drive, ejaculatory function, sexual problems and sexual satisfaction ${ }^{16}$.The investigation of erectile disorders in diabetic patients has focused primarily on organic pathology to the neglect of associated psychological factors. However, there have been few controlled studies that have investigated individual and marital pathology in male diabetic patients or those have assessed the significance of psychological dimensions on the sexual impact of this illness using psychometrically well-established inventories.

Concern has been raised by various studies on the subject of depression with diabetes that requires careful management to improve the quality of life. Berardis et al. ${ }^{17}$ conducted study to identify the cause of erectile dysfunction and quality of life in type 2 diabetic patients. Study involved 1,460 patients enrolled by 114 diabetes outpatient clinics and 112 general practitioners. Patients were asked to complete a questionnaire investigating their ability to achieve and maintain an erection. Overall, $34 \%$ of the patients reported frequent erectile problems, $24 \%$ reported occasional problems, and $42 \%$ reported no erectile problems. After adjusting for patient characteristics, erectile dysfunction was associated with higher levels of diabetes-specific health distress and worse psychological adaptation to diabetes, which were, in turn, related to worse metabolic control. Neuropathy, vascular insufficiency and psychological problems have been implicated in impotence, impaired ejaculation and decreased libido in men and in decreased vaginal lubrication, orgasmic dysfunction and decreased libido in women ${ }^{18}$.

One of the most significant current discussions in diabetes and sexual health is depression disorder that consequence of biochemical and psychological factors ${ }^{19}$. A study conducted by Enzlin et al. to measure the prevalence of sexual dysfunction in patients with diabetes; to describe how descriptive variables, psychological variables, diabetic complications, and sexual dysfunction relate in patients with diabetes; and to illustrate the predictors of sexual dys- function in patients with diabetes ${ }^{20}$. A total of 240 adult type 1 diabetic patients visiting the outpatient diabetes clinic of a university hospital completed questionnaires evaluating psychological adjustment to diabetes and sexual functioning. The result of study demonstrates that sexual dysfunction was reported by $27 \%$ of women and $22 \%$ of men. Study suggests that in men with diabetes, sexual dysfunction is related to somatic and psychological factors, whereas in women with diabetes, psychological factors are more predominant.

Moreover differences in health protective behaviours have been noted between men and women with diseases like diabetes and tuberculosis that include a social component. In these diseases, interactions with others may be influenced by the diseases or individuals with the disease may rely on other to help them be complaint, in the latter case, women have been found to be less complaint in some situations.

Health psychologists aim to change health behaviours for the dual purpose of helping people stay healthy and helping patients adhere to disease treatment regimens. The most important solution is to train physicians and nurses in communicating with patients to emphasize the psychosocial barriers in understanding, remembering and applying effective stratagem for reducing risk factors and making behaviour changes and that will help them to develop suitable means for communicating information related to diagnosis, treatment and prognosis ${ }^{21}$.

Female sexual problems are most strongly associated with psychosocial problems. The strongest association with dyspareunia in female was psychological status, in particular the presence of depression. The proportion of women with dyspareunia increased with increasing depression score. As a result of the impact of diabetes on multiple body systems, women suffer from medical and psychological problems, including sexual dysfunction ${ }^{22}$.

However, a woman's adherence to traditional sex roles may be a hidden barrier to her compliance with a diabetic regimen. The women may not be willing to change her family's life style to accommodate her health needs, may not feel that she has strong support from her family or may be unwilling to discuss her illness with her husband ${ }^{23}$.

Females with type 2 diabetes have double risk of developing abnormal lubrication, decrease sexual desire and painful intercourse compared with non-diabetics. However far too little attention has been given on sexual dysfunction in diabetic women ${ }^{24}$. A controlled study performed to examine the prevalence of sexual problems in women with type 1 diabetes. A total of 120 women with diabetes visiting the outpatient diabetes clinic completed questionnaires evaluating psychological adjustment to diabetes, marital satisfaction, depression, and sexual functioning ${ }^{25}$. The results of study confirm that women with diabetes are clearly at risk for decreased desire and dyspareunia and that especially the arousal phase can be affected. The results also suggest that psychological and not diabetes-related somatic factors are related to sexual dysfunction in women with diabetes. 
Some women's poor health protective behaviour may be explained by research on the effect of sex role stereotyping and sexism on women's behaviour in general, for example women often put other people's needs before their own ${ }^{26}$. They may also be influenced by external factors, such as feeling that they must please their husbands or lose weight to be appealing, even at the expense of their health and wellbeing ${ }^{27}$.

Women with type 2 diabetes experience feelings of depression, anxiety, and anger, which affect their health and overall quality of life ${ }^{28}$. The most common sexual dysfunction in women with diabetes is decreased sexual arousal with slow and inadequate lubrication. Women with diabetes may, however, also experience a decreased sexual desire and more pain on sexual intercourse, whereas problems with orgasm are not more frequent ${ }^{9}$. The research strongly suggests that neurological dysfunctions are linked to many, but not all, cases of erectile failure in diabetic men. For diabetic women, the findings are less cohesive, but suggest that psychological factors may be more important etiologically than physical ones ${ }^{29}$.

Diabetes significantly impairs the sexual performance of diabetic men and women. Contributing factor of sexual function include age and duration of diabetes ${ }^{30}$. Sexual dysfunction needs to be considered in the assessment of both male and female diabetic patients. Furthermore, the close collaboration between dialectologists and psychologists would be fruitful. By good psychosexual counselling patients can converse their shame about asking, buying, and using vaginal lubricants, can help women overcome the painful sexual problems they may be facing. Recent studies suggest that if healthcare providers engage patients in timely conversations, jointly select and implement tailored treatment options and, when necessary, link patients with social support systems to maximize improvements in both physical and psychological aspects of sexual health.

\section{Conclusions}

There are several factors, which have an effect on the psychological aspect of diabetic patients, such as age, gender, place of living, family and social support, motivation, sexual health, life satisfaction, and lifestyle. Social demand of a diabetic patient needs to be taken care. Social requirement regarding health of a person is related to the World Health Organization's statement of Alma Ata (aiming provision of health for all by 2000). Though the statement is not very clear about the definition or specification of health, WHO stresses on a level of health which permits a social and productive life.

First step of meeting the WHO's challenge for health psychologist would be, providing a continuous good research identifying the health risk associated with different behaviours and social conditions. The research data then could be used to create pressure on the policy makers (e.g. politician, economist etc.) who will in turn make policy in an attempt to eliminate conditions such as poverty, unemployment and loneliness. All of which are potent sources of ill health, secondary to or in addition to diabetes. As because many of high risk behaviour of an individual such as food habit, smoking and alcohol consumption are directly related to the development and /or outcome of the treatment protocol for a diabetic patient.

A social or community based approach would help to achieve those goal. Because this will help in understanding the social environment the person is living in, the barrier the person is facing in following or implementing the lifestyle or treatment he or she was prescribed by the service provider (e.g. social stigma about the sexual behaviour or norm of sexual practice in that particular society).

Team work is an essential aspect needed to be considered to evaluate and manage a diabetic patient. The entire member involved in a multidisciplinary team in the treatment/management of diabetes, need education in the psychological aspects of living with a chronic disease like diabetes. Scope of health psychology in the management of diabetes and its complication (e.g. erectile dysfunction) are variable. It can be seen in the application of stress-control procedures, behaviour modification etc. Existing diabetic treatment or management can be enhanced by designing educational programmes and study of the variables that influence self-care behaviour of a diabetic patient. Other aspect would be clarification about process of adaptation in a chronic disease like diabetes and its associated complication such as erectile dysfunction.

\section{Key Points}

- There are strong physical, social, and psychological associations with sexual problems.

- Male sexual problems are most strongly associated with age and physical problems.

- Female sexual problems are most strongly associated with psychosocial problems.

- Effective psychotherapy for sexual problems have a broad impact on health in diabetic population

\section{REFERENCES}

[1] Bethesda. National Institute of Diabetes and Digestive and Kidney Diseases: National diabetes statistics fact sheet: general information and national estimates on diabetes in the United States, U.S. Department of Health and Human Services, National Institutes of Health [Online] 2003. Available from:http://diabetes.niddk.nih.gov/dm/pubs/statistics/index.h tm\#17[Accessed March. 05 2009]

[2] World Health Organization. Diabetes Fact sheet [Online] 2006. Availablefrom:http://www.who.int/mediacentre/factsh eets/fs312/en/ [Accessed Feb. 02 2009]

[3] Ryan CM. Psychological factors and diabetes mellitus: In Textbook of Diabetes. 2nd ed. Pickup J, Williams G, Eds. Oxford, U.K., Blackwell Science;1997

[4] Macfarlane I, Bliss M, Jackson JGL, Williams G.The history of diabetes. In Textbook of Diabetes, 2nd ed. Oxford, U.K., 
Blackwell Science, 01-19; 1997

[5] Enzlin P, Mathieu C, Demytteanere K. Diabetes and Female Sexual Functioning: A State-of-the-Art. Diabetes Spectrum2003, 16(4):256-259

[6] Kolodny RC. Sexual dysfunction in diabetic females. Diabetes 1971, 20:557-559

[7] Isidro ML. Sexual dysfunction in men with type 2 diabetes. Postgrad Med J 2012, 88:152-159

[8] Salonia A, Lanzi R, Scavini M, Pontillo M, Gatti E, Petrella G, Licata G, Nappi, RE, Bosi E, Briganti A, Rigatti P, Montorsi F. Sexual Function and Endocrine Profile in Fertile Women With Type 1 Diabetes. Diabetes Care 2006, 29: 312-316

[9] Enzlin P, Mathieu C, Vanderschueren D, Demyttenaere K. Diabetes mellitus and female sexuality: a review of 25 years' research. Diabet Med 1998, 15:809-815

[10] Guay AT. Sexual dysfunction in the diabetic patient. Int J Impot Res 2001, 13(suppl 5):S47 S50

[11] Jackson G. Sexual dysfunction and diabetes. Int J Clin Pract 2004, 58: 358-62

[12] Bennett P, Weinman J, Spurgeon P. Clinical Health Psychology, Oxford, U.K., Psychology press;1990

[13] Gregoire A. ABC of sexual health: Male sexual problems. BMJ1999, 318:245-247

[14] Hackett GI. Impotence - the most neglected complication of diabetes. Diabetes Research1995,28:75-83

[15] Latini DM, Penson DF, Colwell HH, Lubeck DP, Mehta S, Henning JM, Lue TM. Psychological Impact of Erectile Dysfunction: Validation of a New Health Related Quality of Life Measure for Patients with Erectile Dysfunction. The Journal of Urology2002, 168(5):2086-2091

[16] Burke JP, Jacobson DJ, McGree ME, Nehra A, Roberts RO, Lieber MM, Jacobsen SJ. Diabetes and sexual dysfunction: Result from Olmsted county study of urinary symptoms and health status among men. The journal of urology2007, 177(4):1438-1442

[17] Berardis GD, Franciosi M, Belfigli M. et al.. Erectile Dysfunction and Quality of Life in Type 2 Diabetic Patients: A serious problem too often overlooked. Diabetes Care2002, 25:284-291
[18] Zemel P. Sexual dysfunction in the diabetic patient with hypertension. Amer J of Card1988, 61(16):27-33

[19] Talbot F, Nouwen A. A review of the relationship between depression and diabetes in adults: is there link. Diabetic Care2000, 23(10):1556-1562

[20] Enzlin P, Mathieu C, Bruel AV, Vanderschueren D, Demyttenaere D. Prevalence and Predictors of Sexual Dysfunction in Patients with Type 1 Diabetes. Diabetes Care2003, 26:409-414

[21] Ogden J.A textbook Health Psychology, 3rd ed. Berkshire, England, Open University Press;2004

[22] Grandjean C, Moran PB. The Impact of Diabetes Mellitus on Female Sexual Well-Being. Nursing clinic of n America2007, 42(4):581-592

[23] Martz DM, Handley KB, Eisler RM. The relationship between feminine gender role stress, body image, and eating disorders. Psychology of WomenQuarterly1995, 19:493-508

[24] Sarkadi A, Rosenqvist U. Contraindication in medical encounter: female sexual dysfunction in primary care contact. Family Practice2001, 18(2):161-166

[25] Enzlin P, Mathieu C, Bruel AV, Bosteels J, Vanderschueren D,Demyttenaere D. Sexual Dysfunction in Women with Type 1 Diabetes. Diabetes Care2002,25:672-677

[26] Detzer MJ, Wendt SJ, Soloman LJ, Dorsch E, Geller BM, Friedman J, Hauser H, Flynn BS, and Dorwaldt AL. Barriers to condom use among women attending Planned Parenthood clinics. Women and Health1995, 23:91-102

[27] Johnson M, Elliott BA. Domestic violence among family practice patients in midsized and rural communities. Journal of Family Medicine1997, 44:391-400.

[28] Penckofer S, Ferrans CE, Velsor-Friedrich B, Savoy S. The Psychological Impact of Living with Diabetes: Women's Day-to-Day Experiences.The Diabetes Educator2007, 33(4):680-690

[29] Thomas AM, LoPiccolo J. Sexual functioning in persons with diabetes: Issues in research, treatment, and education. Clinical Psychology Review1994, 14(1):61-86

[30] Fatemi SS, Taghavi SM. Evaluation of sexual function in women with type 2 diabetes mellitus. Diabetes Vasc Dis Res 2009, 6:38-39 